\title{
CONTINUOUS HOMOMORPHISMS OF ARENS-MICHAEL ALGEBRAS
}

\author{
ALEX CHIGOGIDZE
}

Received 3 March 2001 and in revised form 3 July 2001

\begin{abstract}
It is shown that every continuous homomorphism of Arens-Michael algebras can be obtained as the limit of a morphism of certain projective systems consisting of Fréchet algebras. Based on this, we prove that a complemented subalgebra of an uncountable product of Fréchet algebras is topologically isomorphic to the product of Fréchet algebras.
\end{abstract}

2000 Mathematics Subject Classification: 46H05, 46M10.

1. Introduction. Arens-Michael algebras are limits of projective systems of Banach algebras (or, alternatively, closed subalgebras of (uncountable) products of Banach algebras). Quite often, when dealing with a particular ArensMichael algebra, at least one projective system arises naturally (e.g., as a result of certain construction) and, in most cases, it does contain the needed information about its limit. The situation is somewhat different if an Arens-Michael algebra is given arbitrarily and there is no particular projective system associated with it in a canonical way.

Below (Definition 3.2), we introduce the concept of a projective Fréchet system and show (Theorem 3.4) that every continuous homomorphism of ArensMichael algebras can be obtained as the limit homomorphism of certain morphism of cofinal subsystems of the corresponding Fréchet systems. This result applied to the identity homomorphism obviously implies that any ArensMichael algebra has essentially unique Fréchet system associated with it. Consequently, any information about an Arens-Michael algebra is contained in the associated Fréchet system. The remaining problem of restoring this information is, of course, still nontrivial, but sometimes can be successfully handled by using a simple but effective method. This method is based on Proposition 2.5 (applications of Proposition 2.5 in a variety of situations can be found in [1]).

\section{Preliminaries}

2.1. Projective systems and their morphisms. Below, we consider projective systems $\mathscr{S}_{X}=\left\{X_{\alpha}, p_{\alpha}^{\beta}, A\right\}$ consisting of topological algebras $X_{\alpha}, \alpha \in A$, and continuous homomorphisms $p_{\alpha}^{\beta}: X_{\beta} \rightarrow X_{\alpha}, \alpha \leq \beta, \alpha, \beta \in A$ ( $A$ is the directed indexing set of $\mathscr{S}_{X}$ ). The $\operatorname{limit} \lim _{\mathscr{I}_{X}}$ of this system is defined as the closed 
subalgebra of the Cartesian product $\prod\left\{X_{\alpha}: \alpha \in A\right\}$ (with coordinatewise defined operations) consisting of all threads of $\mathscr{S}_{X}$, that is,

$$
\underline{\lim } \mathscr{S}_{X}=\left\{x_{\alpha} \in \prod\left\{X_{\alpha}: \alpha \in A\right\}: p_{\alpha}^{\beta}\left(x_{\beta}\right)=x_{\alpha} \text { for any } \alpha, \beta \in A \text { with } \alpha \leq \beta\right\} .
$$

The $\alpha$ th limit projection $p_{\alpha}: \lim _{\mathscr{l}_{X}} \rightarrow X_{\alpha}, \alpha \in A$, of the system $\mathscr{I}_{X}$ is the restriction (onto $\lim _{\mathscr{S}_{X}}$ ) of the $\alpha$ th natural projection $\pi_{\alpha}: \prod\left\{X_{\alpha}: \alpha \in A\right\} \rightarrow X_{\alpha}$.

If $A^{\prime}$ is a directed subset of the indexing set $A$, then the subsystem $\left\{X_{\alpha}, p_{\alpha}^{\beta}\right.$, $\left.A^{\prime}\right\}$ of $\mathscr{Y}_{X}$ is denoted by $\mathscr{Y}_{X} \mid A^{\prime}$. We refer the reader to $[4,5]$ for general properties of projective systems.

Suppose we are given two projective systems $\mathscr{S}_{X}=\left\{X_{\alpha}, p_{\alpha}^{\beta}, A\right\}$ and $\mathscr{S}_{Y}=$ $\left\{Y_{\gamma}, q_{\gamma}^{\delta}, B\right\}$ consisting of topological algebras $X_{\alpha}, \alpha \in A$, and $Y_{\gamma}, \gamma \in B$. A morphism of the system $\mathscr{S}_{X}$ into the system $\mathscr{S}_{Y}$ is a family $\left\{\varphi,\left\{f_{\gamma}: \gamma \in B\right\}\right\}$, consisting of a nondecreasing function $\varphi: B \rightarrow A$ such that the set $\varphi(B)$ is cofinal in $A$, and of continuous homomorphisms $f_{\gamma}: X_{\varphi(\gamma)} \rightarrow Y_{\gamma}$ defined for all $\gamma \in B$ such that

$$
q_{\gamma}^{\delta} f_{\delta}=f_{\gamma} p_{\varphi(\gamma)}^{\varphi(\delta)}
$$

whenever $\gamma, \delta \in B$ and $\gamma \leq \delta$. In other words, we require (in the above situation) the commutativity of the following diagram:

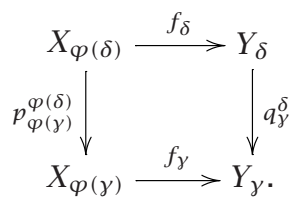

Any morphism

$$
\left\{\varphi,\left\{f_{\gamma}: \gamma \in B\right\}\right\}: \mathscr{S}_{X} \longrightarrow \mathscr{S}_{Y}
$$

induces a continuous homomorphism, called the limit homomorphism of the morphism

$$
\leftrightarrows\left\{\varphi,\left\{f_{\gamma}: \gamma \in B\right\}\right\}: \lim _{\mathscr{Y}_{X}} \longrightarrow \lim \mathscr{S}_{Y} .
$$

To see this, assign to each thread $x=\left\{x_{\alpha}: \alpha \in A\right\}$ of the system $\mathscr{Y}_{X}$ the point $y=\left\{y_{\gamma}: \gamma \in B\right\}$ of the product $\prod\left\{Y_{\gamma}: \gamma \in B\right\}$ by letting

$$
y_{\gamma}=f_{\gamma}\left(x_{\varphi(\gamma)}\right), \quad \gamma \in B .
$$


It is easily seen that the point $y=\left\{y_{\gamma}: \gamma \in B\right\}$ is in fact a thread of the system $\mathscr{S}_{Y}$. Therefore, assigning to $x=\left\{x_{\alpha}: \alpha \in A\right\} \in \lim \mathscr{S}_{X}$ the point $y=\left\{y_{\gamma}: \gamma \in\right.$

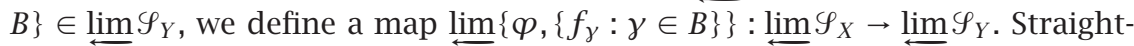
forward verification shows that this map is a continuous homomorphism.

Morphisms of projective systems which arise most frequently in practice are those defined over the same indexing set. In this case, the map $\varphi: A \rightarrow A$ of the definition of morphism is taken to be the identity. Below, we mostly deal with such situations and use the following notation: $\left\{f_{\alpha}: X_{\alpha} \rightarrow Y_{\alpha} ; \alpha \in A\right\}: \mathscr{Y}_{X} \rightarrow \mathscr{S}_{Y}$ or sometimes even a shorter form $\left\{f_{\alpha}\right\}: \mathscr{Y}_{X} \rightarrow \mathscr{Y}_{Y}$.

Proposition 2.1. Let $\mathscr{S}_{Y}=\left\{Y_{\alpha}, q_{\alpha}^{\beta}, A\right\}$ be a projective system and $X$ a topological algebra. Suppose that for each $\alpha \in A$ a continuous homomorphism $f_{\alpha}: X \rightarrow Y_{\alpha}$ is given in such a way that $f_{\alpha}=q_{\alpha}^{\beta} f_{\beta}$ whenever $\alpha, \beta \in A$ and $\alpha \leq \beta$. Then, there exists a natural continuous homomorphism $f: X \rightarrow \lim \mathscr{S}_{Y}$ (the diagonal product $\triangle\left\{f_{\alpha}: \alpha \in A\right\}$ ) satisfying, for each $\alpha \in A$, the condition $f_{\alpha}=q_{\alpha} f$.

Proof. Indeed, we only have to note that $X$, together with its identity map $\operatorname{id}_{X}$, forms the projective system $\mathscr{Y}$. So, the collection $\left\{f_{\alpha}: \alpha \in A\right\}$ is in fact a morphism $\mathscr{S} \rightarrow \mathscr{I}_{Y}$. The rest follows from the definitions given above.

2.2. Arens-Michael algebras. We recall some definitions [3]. A polynormed space $X$ is a topological linear space $X$ furnished with a collection $\left\{\|\cdot\|_{v}, \Lambda\right\}$ of seminorms generating the topology of $X$. This simply means that the collection

$$
\left\{x \in X:\left\|x-x_{0}\right\|_{v}<\epsilon\right\}, \quad x_{0} \in X, v \in \Lambda, \epsilon>0,
$$

forms a subbase of the topology of $X$. A polynormed algebra is a polynormed space $X$ which admits a separately continuous multiplication bioperator $m$ : $X \times X \rightarrow X$. A multinormed algebra is a polynormed algebra such that $\|x y\|_{v} \leq$ $\|x\|_{v} \cdot\|y\|_{v}$ for each $v \in \Lambda$ and any $(x, y) \in X \times X$. Finally, an Arens-Michael algebra is a complete (and Hausdorff) multinormed algebra.

The following result [3, Corollary V.2.19] provides a characterization of Arens-Michael algebras.

THEOREM 2.2. The following conditions are equivalent for a multinormed algebra $X$ :

(a) $X$ is an Arens-Michael algebra;

(b) $X$ is limit of a certain projective system of Banach algebras;

(c) $X$ is topologically isomorphic to a closed subalgebra of the Cartesian product of a certain family of Banach algebras.

2.3. Set-theoretical facts. For the reader's convenience, we present necessary set-theoretic facts. Their complete proofs can be found in [1]. 
Let $A$ be a partially ordered directed set (i.e., for every two elements $\alpha, \beta \in A$ there exists an element $\gamma \in A$ such that $\gamma \geq \alpha$ and $\gamma \geq \beta$ ). We say that a subset $A_{1} \subseteq A$ of $A$ majorates another subset $A_{2} \subseteq A$ of $A$ if for each element $\alpha_{2} \in A_{2}$ there exists an element $\alpha_{1} \in A_{1}$ such that $\alpha_{1} \geq \alpha_{2}$. A subset which majorates $A$ is called cofinal in $A$. A subset of $A$ is said to be a chain if every two elements of it are comparable. The symbol $\sup B$, where $B \subseteq A$, denotes the lower upper bound of $B$ (if such an element exists in $A$ ). Let now $\tau$ be an infinite cardinal number. A subset $B$ of $A$ is said to be $\tau$-closed in $A$ if for each chain $C \subseteq B$, with $|C| \leq \tau$, we have $\sup C \in B$, whenever the element $\sup C$ exists in $A$. Finally, a directed set $A$ is said to be $\tau$-complete if for each chain $C$ of elements of $A$ with $|C| \leq \tau$, there exists an element $\sup C$ in $A$.

The standard example of a $\tau$-complete set can be obtained as follows. For an arbitrary set $A$, let $\exp A$ denote, as usual, the collection of all subsets of $A$. There is a natural partial order on $\exp A: A_{1} \geq A_{2}$ if and only if $A_{1} \supseteq A_{2}$. With this partial order, $\exp A$ becomes a directed set. If we consider only those subsets of the set $A$ which have cardinality $\leq \tau$, then the corresponding subcollection of $\exp A$, denoted by $\exp _{\tau} A$, serves as a basic example of a $\tau$-complete set.

Proposition 2.3. Let $\left\{A_{t}: t \in T\right\}$ be a collection of $\tau$-closed and cofinal subsets of a $\tau$-complete set $A$. If $|T| \leq \tau$, then the intersection $\cap\left\{A_{t}: t \in T\right\}$ is also cofinal (in particular, nonempty) and $\tau$-closed in $A$.

COROLLARY 2.4. For each subset $B$, with $|B| \leq \tau$, of a $\tau$-complete set $A$, there exists an element $\gamma \in A$ such that $\gamma \geq \beta$ for each $\beta \in B$.

Proposition 2.5. Let $A$ be a $\tau$-complete set and $L \subseteq A^{2}$, and suppose that the following three conditions are satisfied.

Existence: for each $\alpha \in A$ there exists $\beta \in A$ such that $(\alpha, \beta) \in L$.

Majorantness: if $(\alpha, \beta) \in L$ and $\gamma \geq \beta$, then $(\alpha, \gamma) \in L$.

$\tau$-closeness: let $\left\{\alpha_{t}: t \in T\right\}$ be a chain in $A$ with $|T| \leq \tau$. If $\left(\alpha_{t}, \gamma\right) \in L$ for some $\gamma \in A$ and each $t \in T$, then $(\alpha, \gamma) \in L$ where $\alpha=\sup \left\{\alpha_{t}: t \in T\right\}$.

Then, the set of all $L$-reflexive elements of $A$ (an element $\alpha \in A$ is $L$-reflexive if $(\alpha, \alpha) \in L)$ is cofinal and $\tau$-closed in $A$.

3. Continuous homomorphisms of Arens-Michael algebras. The following statement is needed in the proof of Theorem 3.4. In the case when all the $X_{\alpha}$ 's are Banach algebras, its proof can be extracted from [3, Proposition 0.1.9] (see also [3, Proof of Proposition V.1.8] and [2, Proof of Proposition 2.1]).

LEMMA 3.1. Let $\mathscr{S}_{X}=\left\{X_{\alpha}, p_{\alpha}^{\beta}, A\right\}$ be a projective system consisting of ArensMichael algebras $X_{\alpha}, \alpha \in A, Y$ a Banach algebra, and $f: \underset{\lim }{\mathscr{S}} \rightarrow Y$ a continuous homomorphism. Suppose that $p_{\alpha}\left(\lim _{9}\right)$ is dense in $X_{\alpha}$ for each $\alpha \in A$. Then, there exist an index $\alpha \in A$ and a continuous homomorphism $f_{\alpha}: X_{\alpha} \rightarrow Y$ such that $f=f_{\alpha} \circ p_{\alpha}$. 
Proof. The continuity of $f$ and the definition of the topology on $\lim \mathscr{S}$ guarantee that there exist an index $\alpha \in A$ and an open subset $V_{\alpha} \subseteq X_{\alpha}$ such that

$$
f\left(p_{\alpha}^{-1}\left(V_{\alpha}\right)\right) \subseteq\{y \in Y:|y| \leq 1\}
$$

where $|\cdot|$ denotes the norm of the Banach space $Y$.

Since $X_{\alpha}$ is an Arens-Michael algebra, $X_{\alpha}$ can be identified with a closed subalgebra of the product $\prod\left\{B_{t}: t \in T\right\}$ of Banach algebras $B_{t}, t \in T$ (Theorem 2.2). Let $\|\cdot\|_{t}$ denote the norm of the Banach space $B_{t}, t \in T$. For $S \subseteq T$, let $\pi_{S}: \prod\left\{B_{t}\right.$ : $t \in T\} \rightarrow \prod\left\{B_{t}: t \in S\right\}$ denote the natural projection onto the corresponding subproduct. If $S \subseteq T$ is a finite subset of $T$, then $\left\|\left\{x_{t}: t \in S\right\}\right\|_{S}=\max \left\{\left\|x_{t}\right\|_{t}\right.$ : $t \in S\}$ for each $\left\{x_{t}: t \in S\right\} \in \prod\left\{B_{t}: t \in S\right\}$.

Since $V_{\alpha}$ is open in $X_{\alpha}$, the definition of the product topology guarantees the existence of a finite subset $S \subseteq T$ and of a number $\epsilon>0$ such that

$$
\left\{x_{\alpha} \in X_{\alpha}:\left\|\pi_{S}\left(x_{\alpha}\right)\right\|_{S} \leq \epsilon\right\} \subseteq V_{\alpha} .
$$

Combining (3.1) and (3.2), we have

$$
f\left(\left\{x \in X:\left\|\pi_{S}\left(p_{\alpha}(x)\right)\right\|_{S} \leq \epsilon\right\}\right) \subseteq\{y \in Y:|y| \leq 1\} .
$$

It then follows that if $x \in X$ and $\left\|\pi_{S}\left(p_{\alpha}(x)\right)\right\|_{S} \leq 1$, then $\left\|\pi_{S}\left(p_{\alpha}(\epsilon x)\right)\right\|_{S}=$ $\epsilon\left\|\pi_{S}\left(p_{\alpha}(x)\right)\right\|_{S} \leq \epsilon$ and consequently

$$
\epsilon|f(x)|=|f(\epsilon x)| \leq 1, \quad \text { that is, } \quad|f(x)| \leq \frac{1}{\epsilon} .
$$

Since

$$
\left\|\pi_{S}\left(p_{\alpha}\left(\frac{x}{\left\|\pi_{S}\left(p_{\alpha}(x)\right)\right\|_{S}}\right)\right)\right\|=\frac{1}{\left\|\pi_{S}\left(p_{\alpha}(x)\right)\right\|_{S}} \cdot\left\|\pi_{S}\left(p_{\alpha}(x)\right)\right\|_{S}=1,
$$

we must have (by (3.4))

$$
\frac{1}{\left\|\pi_{S}\left(p_{\alpha}(x)\right)\right\|_{S}}|f(x)|=\left|f\left(\frac{x}{\left\|\pi_{S}\left(p_{\alpha}(x)\right)\right\|_{S}}\right)\right| \leq \frac{1}{\epsilon}
$$

and hence

$$
|f(x)| \leq \frac{1}{\epsilon}\left\|\pi_{S}\left(p_{\alpha}(x)\right)\right\|_{S}, \quad \text { for each } x \in X .
$$


We now show that the map

$$
h(z)=f\left(p_{\alpha}^{-1}\left(\pi_{S}^{-1}(z) \cap X_{\alpha}\right)\right): \pi_{S}\left(p_{\alpha}(X)\right) \rightarrow Y
$$

is well defined. Assuming the contrary, suppose that for some $z \in \pi_{S}\left(p_{\alpha}(X)\right)$ there exist two points $x_{1}, x_{2} \in p_{\alpha}^{-1}\left(\pi_{S}^{-1}(z) \cap X_{\alpha}\right)$ such that $f\left(x_{1}\right) \neq f\left(x_{2}\right)$. Consequently, $\left|f\left(x_{1}-x_{2}\right)\right| \neq 0$. On the other hand, $\pi_{S}\left(p_{\alpha}\left(x_{1}-x_{2}\right)\right)=\pi_{S}\left(p_{\alpha}\left(x_{1}\right)\right)-$ $\pi_{S}\left(p_{\alpha}\left(x_{2}\right)\right)=z-z=0$. Then, (3.7) implies that

$$
0 \neq\left|f\left(x_{1}-x_{2}\right)\right| \leq \frac{1}{\epsilon}\left\|\pi_{S}\left(p_{\alpha}\left(x_{1}-x_{2}\right)\right)\right\|_{S}=0
$$

This contradiction shows that the map $h: \pi_{S}\left(p_{\alpha}(X)\right) \rightarrow Y$ is indeed well defined. Note that

$$
f=h \circ \pi_{S} \circ p_{\alpha}
$$

which implies that the map $h$ is linear. Next, consider points $z \in \pi_{S}\left(p_{\alpha}(X)\right)$ and $x \in X$ such that $\pi_{S}\left(p_{\alpha}(x)\right)=z$. By (3.7),

$$
|h(z)|=|f(x)| \leq \frac{1}{\epsilon}\left\|\pi_{S}\left(p_{\alpha}(x)\right)\right\|_{S}=\frac{1}{\epsilon}\|z\|_{S} .
$$

This shows that $h$ is bounded and, consequently, continuous. Next, we show that $h: \pi_{S}\left(p_{\alpha}(X)\right) \rightarrow Y$ is multiplicative. Let $\left(x^{\prime}, y^{\prime}\right) \in \pi_{S}\left(p_{\alpha}(X)\right) \times \pi_{S}\left(p_{\alpha}(X)\right)$ and consider a point $(x, y) \in X \times X$ such that $\pi_{S}\left(p_{\alpha}(x)\right)=x^{\prime}$ and $\pi_{S}\left(p_{\alpha}(y)\right)=$ $y^{\prime}$. Then, by (3.10),

$$
\begin{aligned}
h\left(x^{\prime} \cdot y^{\prime}\right) & =h\left(\pi_{S}\left(p_{\alpha}(x)\right) \cdot \pi_{S}\left(p_{\alpha}(y)\right)\right) \\
& =h\left(\pi_{S}\left(p_{\alpha}(x \cdot y)\right)\right) \\
& =f(x \cdot y)=f(x) \cdot f(y) \\
& =h\left(\pi_{S}\left(p_{\alpha}(x)\right)\right) \cdot h\left(\pi_{S}\left(p_{\alpha}(y)\right)\right) \\
& =h\left(x^{\prime}\right) \cdot h\left(y^{\prime}\right) .
\end{aligned}
$$

Since $(Y,|\cdot|)$ is complete, $h$ admits the linear continuous extension

$$
g: \operatorname{cl}_{\prod\left\{B_{t}: t \in S\right\}}\left(\pi_{S}\left(p_{\alpha}(X)\right)\right) \longrightarrow Y .
$$

Since the multiplication on $\prod\left\{B_{t}: t \in T_{f}\right\}$ is jointly continuous, we conclude that $g$ is also multiplicative. Finally, define the map $f_{\alpha}$ as the composition

$$
f_{\alpha}=g \circ\left(\pi_{S} \mid X_{\alpha}\right): X_{\alpha} \rightarrow Y
$$


Obviously, $f_{\alpha}$ is a continuous homomorphism satisfying the required equality $f_{\alpha} \circ p_{\alpha}=f$.

Next, we introduce the concept of projective Fréchet system.

DEFINITION 3.2. Let $\tau \geq \omega$ be a cardinal number. A projective system $\mathscr{P}_{X}=\left\{X_{\alpha}, p_{\alpha}^{\beta}, A\right\}$ consisting of topological algebras $X_{\alpha}$ and continuous homomorphisms $p_{\alpha}^{\beta}: X_{\beta} \rightarrow X_{\alpha}, \alpha \leq \beta, \alpha, \beta \in A$, is a $\tau$-system if

(1) $X_{\alpha}$ is a closed subalgebra of the product of at most $\tau$ Banach algebras, $\alpha \in A$;

(2) the indexing set $A$ is $\tau$-complete;

(3) if $\left\{\alpha_{\gamma}: \gamma \in \tau\right\}$ is an increasing chain of elements in $A$ with $\alpha=\sup \left\{\alpha_{\gamma}\right.$ : $\gamma \in \tau\}$, then the diagonal product (see Proposition 2.1)

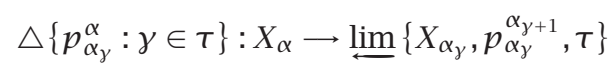

is a topological isomorphism;

(4) $p_{\alpha}(X)$ is dense in $X_{\alpha}$ for each $\alpha \in A$.

Fréchet systems are defined as projective $\omega$-systems.

Proposition 3.3. Every Arens-Michael algebra $X$ can be represented as the limit of a projective Fréchet system $\mathscr{S}_{X}=\left\{X_{A}, p_{A}^{B}\right.$, $\left.\exp _{\omega} T\right\}$. Conversely, the limit of a projective Fréchet system is an Arens-Michael algebra.

Proof. By Theorem 2.2, $X$ can be identified with a closed subalgebra of the product $\prod\left\{X_{t}: t \in T\right\}$ of some collection of Banach algebras. If $|T| \leq \omega$, then $X$ itself is a Fréchet algebra and therefore our statement is trivially true. If $|T|>$ $\omega$, then consider the set $\exp _{\omega} T$ of all countable subsets of $T$. Clearly, $\exp _{\omega} T$ is $\omega$-complete set (see Section 2.3). For each $A \in \exp _{\omega} T$, let $X_{A}=\operatorname{cl} \pi_{A}(X)$ (closure is taken in $\prod\left\{X_{t}: t \in A\right\}$ ), where

$$
\pi_{A}: \prod\left\{X_{t}: t \in T\right\} \rightarrow \prod\left\{X_{t}: t \in A\right\}
$$

denotes the natural projection onto the corresponding subproduct. Also let $p_{A}^{B}=\pi_{A}^{B} \mid X_{B}$, where

$$
\pi_{A}^{B}: \prod\left\{X_{t}: t \in B\right\} \rightarrow \prod\left\{X_{t}: t \in A\right\}
$$

is the natural projection, $A, B \in \exp _{\omega} T, A \leq B$. The straightforward verification shows that $\mathscr{S}_{X}=\left\{X_{A}, p_{A}^{B}\right.$, $\left.\exp _{\omega} T\right\}$ is indeed a projective Fréchet system such that $\lim _{X} \mathscr{S}_{X}=X$.

Conversely, let $\mathscr{S}_{X}=\left\{X_{\alpha}, p_{\alpha}^{\beta}, A\right\}$ be a projective Fréchet system. Clearly, $\longleftarrow \mathscr{I}_{X}$ can be identified with a closed subalgebra of the product $\prod\left\{X_{\alpha}: \alpha \in A\right\}$ (see Section 2.1). Each $X_{\alpha}, \alpha \in A$, can obviously be identified with a closed subalgebra of the product $\prod\left\{B_{n}^{\alpha}: n \in T_{\alpha}\right\}$ of a countable collection of Banach algebras $B_{n}^{\alpha}$. Then $\varliminf^{\lim } \mathscr{Y}_{X}$, as a closed subalgebra of the product $\prod\left\{\prod\left\{B_{n}^{\alpha}: n \in\right.\right.$ $\left.\left.T_{\alpha}\right\}: a \in A\right\}$, according to Theorem 2.2, is an Arens-Michael algebra. 
The following theorem is one of our main results.

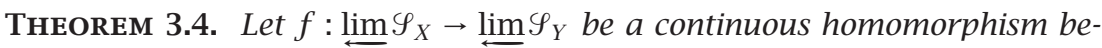
tween the limits of two projective Fréchet systems $\mathscr{S}_{X}=\left\{X_{\alpha}, p_{\alpha}^{\beta}, A\right\}$ and $\mathscr{S}_{Y}=$ $\left\{Y_{\alpha}, q_{\alpha}^{\beta}, A\right\}$ with the same indexing set $A$. Then, there exist a cofinal and $\omega$-closed subset $B_{f}$ of $A$ and a morphism

$$
\left\{f_{\alpha}: X_{\alpha} \longrightarrow Y_{\alpha}, B_{f}\right\}: \mathscr{Y}_{X}\left|B_{f} \longrightarrow \mathscr{Y}_{Y}\right| B_{f}
$$

consisting of continuous homomorphisms $f_{\alpha}: X_{\alpha} \rightarrow Y_{\alpha}, \alpha \in B_{f}$, such that $f=$ $\stackrel{\lim }{\longleftarrow}\left\{f_{\alpha}: B_{f}\right\}$.

If, in particular, $\lim _{\varphi_{X}}$ and $\lim _{\varphi_{Y}}$ are topologically isomorphic, then $\mathscr{S}_{X}=$ $\left\{X_{\alpha}, p_{\alpha}^{\beta}, A\right\}$ and $\mathscr{S}_{Y}=\left\{Y_{\alpha}, q_{\alpha}^{\beta}, A\right\}$ contain isomorphic cofinal and $\omega$-closed subsystems.

PRoof. We perform the spectral search by means of the following relation

$$
\begin{aligned}
& L=\left\{(\alpha, \beta) \in A^{2}: \alpha \leq \beta\right. \text { and there exists } \\
&\text { a continuous homomorphism } \left.f_{\alpha}^{\beta}: X_{\beta} \longrightarrow Y_{\alpha} \text { such that } f_{\alpha}^{\beta} p_{\beta}=q_{\alpha} f\right\} .
\end{aligned}
$$

We verify the conditions of Proposition 2.5.

EXISTENCE CONDITION. By assumption, $Y_{\alpha}$ is a Fréchet algebra. Therefore, $Y_{\alpha}$ can be identified with a closed subspace of a countable product $\prod\left\{B_{n}: n \in\right.$ $\omega\}$ of Banach algebras. Let $\pi_{n}: \prod\left\{B_{m}: m \in \omega\right\} \rightarrow B_{n}$ denote the $n$th natural projection. For each $n \in \omega$, by Lemma 3.1, there exist an index $\beta_{n} \in A$ and a continuous homomorphism $f_{\beta_{n}}: X_{\beta_{n}} \rightarrow B_{n}$ such that $\pi_{n} q_{\alpha} f=f_{\beta_{n}} p_{\beta_{n}}$. By Corollary 2.4, there exists an index $\beta \in A$ such that $\beta \geq \beta_{n}$ for each $n$. Without loss of generality, we may assume that $\beta \geq \alpha$. Let $f_{n}=f_{\beta_{n}} p_{\beta_{n}}^{\beta}, n \in \omega$. Next, consider the diagonal product

$$
f_{\alpha}^{\beta}=\triangle\left\{f_{n}: n \in \omega\right\}: X_{\beta} \rightarrow \prod\left\{B_{n}: n \in \omega\right\}
$$

Obviously, $f_{\alpha}^{\beta} p_{\beta}=q_{\alpha} f$. It only remains to show that $f_{\alpha}^{\beta}\left(X_{\beta}\right) \subseteq Y_{\alpha}$. First, observe that $f_{\alpha}^{\beta}\left(p_{\beta}(X)\right) \subseteq Y_{\alpha}$. Indeed, let $x \in X$. Then,

$$
\begin{aligned}
f_{\alpha}^{\beta}\left(p_{\beta}(x)\right) & =\left\{f_{n}\left(p_{\beta}(x)\right): n \in \omega\right\} \\
& =\left\{f_{\beta_{n}}\left(p_{\beta_{n}}^{\beta}\left(p_{\beta}(x)\right)\right): n \in \omega\right\} \\
& =\left\{f_{\beta_{n}}\left(p_{\beta_{n}}(x)\right): n \in \omega\right\} \\
& =\left\{\pi_{n}\left(q_{\alpha}(f(x))\right): n \in \omega\right\} \\
& =q_{\alpha}(f(x)) \in Y_{\alpha} .
\end{aligned}
$$


Finally,

$$
\begin{aligned}
f_{\alpha}^{\beta}\left(X_{\beta}\right) & =f_{\alpha}^{\beta}\left(\operatorname{cl}_{X_{\beta}} p_{\beta}(X)\right) \subseteq \operatorname{cl}_{\prod\left\{B_{n}: n \in \omega\right\}} f_{\alpha}^{\beta}\left(p_{\beta}(X)\right) \subseteq \operatorname{cl}_{\prod\left\{B_{n}: n \in \omega\right\}} Y_{\alpha} \\
& =Y_{\alpha} .
\end{aligned}
$$

MAJORANTNESS CONDITION. The verification of this condition is trivial. Indeed, it suffices to consider the composition $f_{\alpha}^{\gamma}=f_{\alpha}^{\beta} p_{\beta}^{\gamma}$.

$\omega$-CLOSENESS CONDITION. Suppose that for some countable chain $C=\left\{\alpha_{n}\right.$ : $n \in \omega\}$ in $A$ with $\alpha=\sup C$ and for some $\beta \in A$ with $\beta \geq \alpha$, the maps $f_{\alpha_{n}}^{\beta}: X_{\beta} \rightarrow$ $Y_{\alpha_{n}}$ have already been defined in such a way that $f_{\alpha_{n}}^{\beta} p_{\beta}=q_{\alpha_{n}} f$ for each $n \in \omega$ (in other words, $\left(\alpha_{n}, \beta\right) \in L$ for each $\left.n \in \omega\right)$. Next, consider the composition

$$
f_{\alpha}^{\beta}=i^{-1} \circ \triangle\left\{f_{\alpha_{n}}^{\beta}: n \in \omega\right\}: X_{\beta} \longrightarrow Y_{\alpha},
$$

where $i: Y_{\alpha} \rightarrow \underline{\lim }\left\{Y_{\alpha_{n}}, q_{\alpha_{n}}^{\alpha_{n+1}}, \omega\right\}$ is the topological isomorphism indicated in condition (3) of Definition 3.2. Observe that for each $x \in X$,

$$
\begin{aligned}
f_{\alpha}^{\beta}\left(p_{\beta}(x)\right) & =i^{-1}\left(\triangle\left\{f_{\alpha_{n}}^{\beta}: n \in \omega\right\}\right)\left(p_{\beta}(x)\right) \\
& =i^{-1}\left(\triangle\left\{q_{\alpha_{n}}: n \in \omega\right\}\right)(f(x)) \\
& =i^{-1}\left(\triangle\left\{q_{\alpha_{n}}^{\alpha}: n \in \omega\right\}\right)\left(q_{\alpha}(f(x))\right) \\
& =\left(\triangle\left\{q_{\alpha_{n}}^{\alpha}: n \in \omega\right\}\right)^{-1}\left(\triangle\left\{q_{\alpha_{n}}^{\alpha}: n \in \omega\right\}\right)\left(q_{\alpha}(f(x))\right) \\
& =q_{\alpha}(f(x)) .
\end{aligned}
$$

This shows that $(\alpha, \beta) \in L$ and finishes the verification of the $\omega$-closeness condition.

Now, denote by $B_{f}$ the set of all $L$-reflexive elements in $A$. By Proposition 2.5, $B_{f}$ is a cofinal and $\omega$-closed subset of $A$. One can easily see that the $L$-reflexivity of an element $\alpha \in A$ is equivalent to the existence of a continuous homomorphism $f_{\alpha}=f_{\alpha}^{\alpha}: X_{\alpha} \rightarrow Y_{\alpha}$ satisfying the equality $f_{\alpha} p_{\alpha}=q_{\alpha} f$. Consequently, the collection $\left\{f_{\alpha}: \alpha \in B_{f}\right\}$ is a morphism of the cofinal and $\omega$-closed subspectrum $\mathscr{T}_{X} \mid B_{f}$ of the spectrum $\mathscr{S}_{X}$ into the cofinal and $\omega$-closed subspectrum $\mathscr{S}_{Y} \mid B_{f}$ of the spectrum $\mathscr{S}_{Y}$. It only remains to remark that the original map $f$ is induced by the constructed morphism. This finishes the proof of the first part of our theorem.

The second part of this theorem can be obtained from the first part as fol-

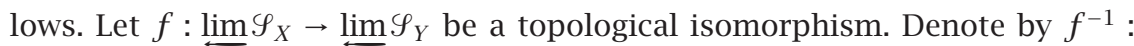
$\longleftarrow \mathscr{l}_{Y} \rightarrow \underset{\lim }{\mathscr{S}_{X}}$ the inverse of $f$. By the first part proved above, there exist a cofinal and $\omega$-closed subset $B_{f}$ of $A$ and a morphism

$$
\left\{f_{\alpha}: X_{\alpha} \longrightarrow Y_{\alpha}: \alpha \in B_{f}\right\}: \mathscr{Y}_{X}\left|B_{f} \longrightarrow \mathscr{Y}_{Y}\right| B_{f}
$$


such that $f=\lim _{\{}\left\{f_{\alpha}: \alpha \in B_{f}\right\}$. Similarly, there exist a cofinal and $\omega$-closed subset $B_{f^{-1}}$ of $A$ and a morphism

$$
\left\{g_{\alpha}: Y_{\alpha} \longrightarrow X_{\alpha}: \alpha \in B_{f^{-1}}\right\}: \mathscr{Y}_{Y}\left|B_{f^{-1}} \longrightarrow \mathscr{Y}_{X}\right| B_{f^{-1}}
$$

such that $f^{-1}=\lim _{2}\left\{g_{\alpha}: \alpha \in B_{f^{-1}}\right\}$.

By Proposition 2.3, the set $B=B_{f} \cap B_{f^{-1}}$ is still cofinal and $\omega$-closed in $A$. Therefore, in order to complete the proof, it suffices to show that for each $\alpha \in B$ the map $f_{\alpha}: X_{\alpha} \rightarrow Y_{\alpha}$ is a topological isomorphism. Indeed, take a point $x_{\alpha} \in p_{\alpha}\left(\lim _{\mathscr{I}_{X}}\right) \subseteq X_{\alpha}$. Also choose a point $x \in \lim _{\mathscr{S}_{X}}$ such that $x_{\alpha}=p_{\alpha}(x)$. Then,

$$
g_{\alpha} f_{\alpha}\left(x_{\alpha}\right)=g_{\alpha} f_{\alpha} p_{\alpha}(x)=g_{\alpha} q_{\alpha} f(x)=p_{\alpha} f^{-1} f(x)=p_{\alpha}(x)=x_{\alpha} .
$$

This proves that $g_{\alpha} f_{\alpha} \mid p_{\alpha}\left(\underline{\lim }^{\mathscr{S}_{X}}\right)=\operatorname{id}_{p_{\alpha}\left(\lim _{\longleftarrow} \varphi_{X}\right)}$. Similar considerations show that $f_{\alpha} g_{\alpha} \mid q_{\alpha}\left(\lim _{\mathcal{I}_{Y}}\right)=\operatorname{id}_{q_{\alpha}\left(\lim _{\longleftarrow} \varphi_{Y}\right)}$ for each $\alpha \in B$. Since $p_{\alpha}\left(\varliminf_{Y}^{\lim } \mathscr{S}_{X}\right)$ is dense in $X_{\alpha}$ and $q_{\alpha}\left(\lim _{\mathscr{S}_{Y}}\right)$ is dense in $Y_{\alpha}$ (Definition 3.2, condition (4)), it follows that $g_{\alpha} f_{\alpha} \mid X_{\alpha}=\operatorname{id}_{X_{\alpha}}$ and $f_{\alpha} g_{\alpha} \mid Y_{\alpha}=\operatorname{id}_{Y_{\alpha}}$. It is now clear that $f_{\alpha}, \alpha \in B$, is a topological isomorphism (whose inverse is $g_{\alpha}$ ).

REMARK 3.5. A similar statement remains true (with the identical proof) for projective $\tau$-systems for any cardinal number $\tau>\omega$.

REMARK 3.6. Theorem 3.4 is false for countable projective systems. Indeed, consider the following two projective sequences

$$
\mathscr{S}_{\text {even }}=\left\{\mathbb{C}^{2 n}, \pi_{2 n}^{2(n+1)}, \omega\right\}, \quad \mathscr{S}_{\text {odd }}=\left\{\mathbb{C}^{2 n+1}, \pi_{2 n+1}^{2(n+1)+1}, \omega\right\} \text {, }
$$

where

$$
\pi_{2 n}^{2(n+1)}: \mathbb{C}^{2 n} \times \mathbb{C}^{2} \longrightarrow \mathbb{C}^{2 n}, \quad \pi_{2 n+1}^{2(n+1)+1}: \mathbb{C}^{2 n+1} \times \mathbb{C}^{2} \longrightarrow \mathbb{C}^{2 n+1}
$$

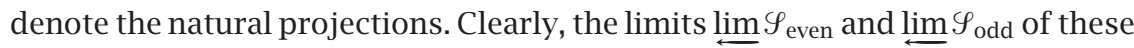
projective systems are topologically isomorphic (both are topologically isomorphic to the countable infinite power $\mathbb{C}^{\omega}$ of $\mathbb{C}$ ), but $\mathscr{S}_{\text {even }}$ and $\mathscr{S}_{\text {odd }}$ do not contain isomorphic cofinal subsystems.

COROLLARY 3.7. Let $\mathscr{S}_{X}=\left\{X_{\alpha}, p_{\alpha}^{\beta}, A\right\}$ be a projective Fréchet system. If $\lim \mathscr{}$ is a Fréchet algebra, then there exists an index $\alpha \in A$ such that the $\beta$ th limit projection $p_{\beta}: \lim \mathscr{S} \rightarrow X_{\beta}$ is a topological isomorphism for each $\beta \geq \alpha$.

Proof. Consider a trivial projective Fréchet system $\mathscr{S}^{\prime}=\left\{X_{\alpha}, q_{\alpha}^{\beta}, A\right\}$, where $X_{\alpha}=\longleftarrow \lim _{\varphi}$ and $q_{\alpha}^{\beta}=\operatorname{id}_{\lim \varphi}$ for each $\alpha, \beta \in A$. By Theorem 3.4 (applied to the identity homomorphism $f=\operatorname{id}_{\lim \varphi}$ ), there exist an index $\alpha \in A$ and a continu-

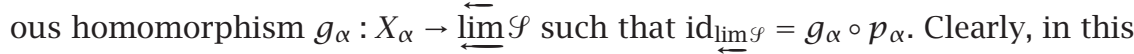
situation, $p_{\alpha} \mid \longleftarrow \mathscr{\operatorname { l i m }} \mathscr{Y}: \lim \mathscr{S} \rightarrow X_{\alpha}$ is an embedding with a closed image. But this 
image $p_{\alpha}(\lim \mathscr{Y})$ is dense in $X_{\alpha}$ (Definition 3.2, condition (4)). Therefore, $p_{\alpha}$ (and, consequently $p_{\beta}$ for any $\beta \geq \alpha$ ) is a topological isomorphism.

COROLLARY 3.8. Suppose that $X$ is a Fréchet subalgebra of an uncountable product $\prod\left\{B_{t}: t \in T\right\}$ of Fréchet (Banach) algebras, then there exists a countable subset $T_{X}$ of the indexing set $T$ such that the restriction $\pi_{T_{X}} \mid X: X \rightarrow \pi_{T_{X}}(X)$ of the natural projection $\pi_{T_{X}}: \prod\left\{B_{t}: t \in T\right\} \rightarrow \prod\left\{B_{t}: t \in T_{X}\right\}$ is a topological isomorphism.

3.1. Arens-Michael $*$-algebras. The concept of projective Fréchet system can naturally be adjusted to handle variety of situations. Below it will always be completely clear in what content this concept is being used. We consider Arens-Michael $*$-algebras, that is, Arens-Michael algebras with a continuous involution. It is known that every such an algebra can be identified with a closed *-subalgebra of the product of Banach $*$-algebras (see, e.g., [3, Proposition V.3.41]). Therefore, one can obtain an alternative description of such algebras as limits of projective systems consisting of Banach $*$-algebras and continuous *-homomorphisms (compare with Theorem 2.2). This, as in Proposition 3.3, leads us to the conclusion recorded in the following proposition.

Proposition 3.9. Every Arens-Micheal $*$-algebra $X$ can be represented as the limit of a projective Fréchet system $\mathscr{S}_{X}=\left\{X_{\alpha}, p_{\alpha}^{\beta}, A\right\}$ consisting of Fréchet $*$-algebras $X_{\alpha}, \alpha \in A$ and continuous $*$-homomorphisms $p_{\alpha}^{\beta}: X_{\beta} \rightarrow X_{\alpha}, \alpha \leq \beta$, $\alpha, \beta \in A$. Conversely, the limit of any such projective Fréchet system is an ArensMichael $*$-algebra.

The analog of Theorem 3.4 is also true.

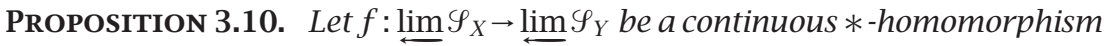
between the limits of two projective Fréchet systems $\mathscr{S}_{X}=\left\{X_{\alpha}, p_{\alpha}^{\beta}, A\right\}$ and $\mathscr{S}_{Y}=$ $\left\{Y_{\alpha}, q_{\alpha}^{\beta}, A\right\}$, consisting of Fréchet $*$-algebras and continuous $*$-homomorphisms and having the same indexing set $A$. Then, there exist a cofinal and $\omega$-closed subset $B_{f}$ of $A$ and a morphism

$$
\left\{f_{\alpha}: X_{\alpha} \longrightarrow Y_{\alpha}, B_{f}\right\}: \mathscr{Y}_{X}\left|B_{f} \longrightarrow \mathscr{Y}_{Y}\right| B_{f}
$$

consisting of continuous $*$-homomorphisms $f_{\alpha}: X_{\alpha} \rightarrow Y_{\alpha}, \alpha \in B_{f}$, such that $f=\lim _{\{}\left\{f_{\alpha} ; B_{f}\right\}$.

If, in particular, $\lim _{\mathscr{S}_{X}}$ and $\lim _{\mathscr{S}_{Y}}$ are topologically $*$-isomorphic, then $\mathscr{S}_{X}=$ $\left\{X_{\alpha}, p_{\alpha}^{\beta}, A\right\}$ and $\mathscr{S}_{Y}=\left\{Y_{\alpha}, q_{\alpha}^{\beta}, A\right\}$ contain isomorphic cofinal and $\omega$-closed subsystems.

Proof. By Theorem 3.4, there exists a cofinal and $\omega$-closed subset $B_{f}$ of $A$ and a morphism

$$
\left\{f_{\alpha}: X_{\alpha} \longrightarrow Y_{\alpha}, B_{f}\right\}: \mathscr{Y}_{X}\left|B_{f} \longrightarrow \mathscr{Y}_{Y}\right| B_{f}
$$


consisting of continuous homomorphisms $f_{\alpha}: X_{\alpha} \rightarrow Y_{\alpha}, \alpha \in B_{f}$, such that

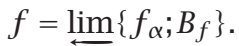

We show that $f_{\alpha}, \alpha \in B_{f}$, is actually a $*$-homomorphism. Indeed, let $x_{\alpha} \in$ $p_{\alpha}(X)$ and $x \in X$ such that $p_{\alpha}(x)=x_{\alpha}$. Then,

$$
\begin{aligned}
f_{\alpha}\left(x_{\alpha}^{*}\right) & =f_{\alpha}\left(p_{\alpha}(x)^{*}\right)=f_{\alpha}\left(p_{\alpha}\left(x^{*}\right)\right) \\
& =q_{\alpha}\left(f\left(x^{*}\right)\right)=q_{\alpha}\left(f(x)^{*}\right) \\
& =q_{\alpha}(f(x))^{*}=f_{\alpha}\left(p_{\alpha}(x)\right)^{*} \\
& =f_{\alpha}\left(x_{\alpha}\right)^{*} .
\end{aligned}
$$

4. Complemented subalgebras of uncountable products of Fréchet algebras. In this section, we show (Theorem 4.2) that the complemented subalgebras of (uncountable) products of Fréchet algebras are products of Fréchet algebras. We begin with the following lemma.

LEMMA 4.1. Let $p: X \rightarrow Y$ be a surjective continuous homomorphism of topological algebras and suppose that $X$ is a closed subalgebra of the product $Y \times B$, where $B$ is a topological algebra. Assume also that there exists a continuous homomorphism $r: Y \times B \rightarrow X$ satisfying the following conditions:

(i) $p r=\pi_{Y}$, where $\pi_{Y}: Y \times B \rightarrow Y$ denotes the natural projection;

(ii) $r(x)=x$ for each $x \in X$.

Then, there exists a topological isomorphism $h: X \rightarrow Y \times \operatorname{ker} p$ such that $\pi_{Y} h=p$.

Proof. If $x \in X$, then

$$
\begin{aligned}
p(x-r(p(x), 0)) & =p(x)-p(r(p(x), 0)) \\
& \stackrel{\text { by (i) }}{=} p(x)-\pi_{Y}(p(x), 0) \\
& =p(x)-p(x) \\
& =0 .
\end{aligned}
$$

This shows that the formula

$$
h(x)=(p(x), x-r(p(x), 0)), \quad x \in X,
$$

defines a continuous linear map $h: X \rightarrow Y \times \operatorname{ker} p$. Moreover, $h$ is a topological isomorphism between $X$ and $Y \times \operatorname{ker} p$ considered as topological vector spaces (to see this observe that the continuous and linear map $g: Y \times \operatorname{ker} p \rightarrow X$ defined by letting $g(y, x)=r(y, 0)+x$ for each $(y, x) \in Y \times \operatorname{ker} p$ has the following properties: $g \circ h=\operatorname{id}_{X}$ and $h \circ g=\operatorname{id}_{Y \times \operatorname{ker} p}$ ). We now show that $h$ is an isomorphism of the category of topological algebras as well.

Let $x_{1}, x_{2} \in X$. We need to show that $h\left(x_{1}\right) \cdot h\left(x_{2}\right)=h\left(x_{1} \cdot x_{2}\right)$. Since $X \subseteq$ $Y \times B$, we can write $x_{i}=\left(a_{i}, b_{i}\right)$, where $a_{i} \in Y$ and $b_{i} \in B, i=1,2$. Observe that since $x_{i} \in X$, it follows from (ii) that $r\left(x_{i}\right)=x_{i}$. Consequently, by (i), 


$$
\begin{aligned}
& p\left(x_{i}\right)=p\left(r\left(x_{i}\right)\right)=\pi_{Y}\left(x_{i}\right)=\pi_{Y}\left(a_{i}, b_{i}\right)=a_{i} \text {. Then, } \\
& \quad h\left(x_{i}\right)=\left(p\left(x_{i}\right), x_{i}-r\left(p\left(x_{i}\right), 0\right)\right)=\left(a_{i},\left(a_{i}, b_{i}\right)-r\left(a_{i}, 0\right)\right), \quad i=1,2 .
\end{aligned}
$$

Consequently,

$$
\begin{aligned}
h\left(x_{1}\right) \cdot h\left(x_{2}\right) & =\left(a_{1},\left(a_{1}, b_{1}\right)-r\left(a_{1}, 0\right)\right) \cdot\left(a_{2},\left(a_{2}, b_{2}\right)-r\left(a_{2}, 0\right)\right) \\
& =\left(a_{1} \cdot a_{2},\left[\left(a_{1}, b_{1}\right)-r\left(a_{1}, 0\right)\right] \cdot\left[\left(a_{2}, b_{2}\right)-r\left(a_{2}, 0\right)\right]\right) \\
& =\left(a_{1} \cdot a_{2},\left[r\left(a_{1}, b_{1}\right)-r\left(a_{1}, 0\right)\right] \cdot\left[r\left(a_{2}, b_{2}\right)-r\left(a_{2}, 0\right)\right]\right) \\
& =\left(a_{1} \cdot a_{2}, r\left(0, b_{1}\right) \cdot r\left(0, b_{2}\right)\right)=\left(a_{1} \cdot a_{2}, r\left[\left(0, b_{1}\right) \cdot\left(0, b_{2}\right)\right]\right) \\
& =\left(a_{1} \cdot a_{2}, r\left(0, b_{1} \cdot b_{2}\right)\right)=\left(a_{1} \cdot a_{2}, r\left[\left(a_{1} \cdot a_{2}, b_{1} \cdot b_{2}\right)-\left(a_{1} \cdot a_{2}, 0\right)\right]\right) \\
& =\left(a_{1} \cdot a_{2}, r\left(a_{1} \cdot a_{2}, b_{1} \cdot b_{2}\right)-r\left(a_{1} \cdot a_{2}, 0\right)\right) \\
& =\left(a_{1} \cdot a_{2},\left(a_{1} \cdot a_{2}, b_{1} \cdot b_{2}\right)-r\left(a_{1} \cdot a_{2}, 0\right)\right) \\
& =h\left(a_{1} \cdot a_{2}, b_{1} \cdot b_{2}\right) \\
& =h\left(\left(a_{1}, b_{1}\right) \cdot\left(a_{2}, b_{2}\right)\right) \\
& =h\left(x_{1} \cdot x_{2}\right) .
\end{aligned}
$$

This shows that $h$ is a homomorphism and, consequently, a topological isomorphism as required.

For algebras with trivial multiplication, the following theorem is contained in [2].

THEOREM 4.2. A complemented subalgebra of the product of uncountable family of Fréchet algebras is topologically isomorphic to the product of Fréchet algebras. More formally, if $X$ is a complemented subalgebra of the product $\prod\left\{B_{t}: t \in T\right\}$ of Fréchet algebras $B_{t}, t \in T$, then $X$ is topologically isomorphic to the product $\prod\left\{F_{j}: j \in J\right\}$, where $F_{j}$ is a complemented subalgebra of the product $\prod\left\{B_{t}: t \in T_{j}\right\}$ with $\left|T_{j}\right|=\omega$ for each $j \in J$.

Proof. Let $X$ be a complemented subalgebra of the uncountable product $B=\prod\left\{B_{t}: t \in T\right\}$ of Fréchet algebras $B_{t}, t \in T$, where $T$ is an indexing set with $|T|=\tau>\omega$. There exists a continuous homomorphism $r: B \rightarrow X$ such that $r(x)=x$ for each $x \in X$. A subset $S \subseteq T$ is called $r$-admissible if $\pi_{S}(r(z))=$ $\pi_{S}(z)$ for each point $z \in \pi_{S}^{-1}\left(\pi_{S}(X)\right)$.

ClaIm 1. The union of an arbitrary family of $r$-admissible sets is $r$-admissible.

This derives from the fact that for $x, y \in B, \pi_{S}(x)=\pi_{S}(y)$ if and only if $\pi_{t}(x)=\pi_{t}(y)$ for each $t \in S$.

ClAIM 2. If $S \subseteq T$ is $r$-admissible, then $\pi_{S}(X)$ is a closed subalgebra of $B_{S}=\prod\left\{B_{t}: t \in S\right\}$. 
Indeed, let $i_{S}: B_{S} \rightarrow B$ be the canonical section of $\pi_{S}$ (this means that $i_{S}=$ $\left.\operatorname{id}_{B_{S}} \triangle \mathbf{0}: B_{S} \rightarrow B_{S} \times B_{T-S}=B\right)$. Consider a continuous linear map $r_{S}=\pi_{S} \circ r \circ i_{S}:$ $B_{S} \rightarrow \pi_{S}(X)$. Obviously, $i_{S}(y) \in \pi_{S}^{-1}\left(\pi_{S}(X)\right)$ for any point $y \in \pi_{S}(X)$. Since $S$ is $r$-admissible, the latter implies that

$$
y=\pi_{S}\left(i_{S}(y)\right)=\pi_{S}\left(r\left(i_{S}(y)\right)\right)=r_{S}(y) .
$$

This shows that $\pi_{S}(X)$ is closed in $B_{S}$.

ClaIm 3. Let $S$ and $R$ be $r$-admissible subsets of $T$ and $S \subseteq R \subseteq T$. Then, the map $\pi_{S}^{R}: \pi_{R}(X) \rightarrow \pi_{S}(X)$ is topologically isomorphic to the natural projection $\pi: \pi_{S}(X) \times \operatorname{ker}\left(\pi_{S}^{R} \mid \pi_{R}(X)\right) \rightarrow \pi_{S}(X)$.

Obviously, $\pi_{R}(X) \subseteq \pi_{S}(X) \times B_{R-S} \subseteq B_{R}=B_{S} \times B_{R-S}$. Consider the map $i_{R}=$ $\operatorname{id}_{B_{R}} \triangle \mathbf{0}: B_{R} \rightarrow B_{R} \times B_{T-R}=B$. Also, let $r_{R}=\pi_{R} \circ r \circ i_{R}: B_{R} \rightarrow \pi_{R}(X)$.

Observe that $\pi_{S}^{R} \circ r_{R}\left|\left(\pi_{S}(X) \times B_{R-S}\right)=\pi_{S}^{R}\right|\left(\pi_{S}(X) \times B_{R-S}\right)$. Indeed, if $x \in$ $\pi_{S}(X) \times B_{R-S}$, then $i_{R}(x) \in \pi_{S}^{-1}\left(\pi_{S}(X)\right)$. Since $S$ is $r$-admissible, we have $\pi_{S}\left(r\left(i_{R}(x)\right)\right)=\pi_{S}\left(i_{R}(x)\right)$. Consequently,

$$
\begin{aligned}
\pi_{S}^{R}\left(r_{R}(x)\right) & =\pi_{S}^{R}\left(\pi_{R}\left(r\left(i_{R}(x)\right)\right)\right)=\pi_{S}\left(r\left(i_{R}(x)\right)\right) \\
& =\pi_{S}\left(i_{R}(x)\right)=\pi_{S}^{R}\left(\pi_{R}\left(i_{R}(x)\right)\right)=\pi_{S}^{R}(x) .
\end{aligned}
$$

Next, observe that $r_{R}(x)=x$ for any point $x \in \pi_{R}(X)$. Indeed, since $R$ is $r$-admissible and since $i_{R}(x) \in \pi_{R}^{-1}\left(\pi_{R}(X)\right)$, we have

$$
r_{R}(x)=\pi_{R}\left(r\left(i_{R}(x)\right)\right)=\pi_{R}\left(i_{R}(x)\right)=x .
$$

Application of Lemma 4.1 (with $X=\pi_{R}(X), Y=\pi_{S}(X), B=B_{R-S}, p=$ $\pi_{S}^{R} \mid \pi_{R}(X)$, and $\left.r=r_{R}\right)$ finishes the proof of Claim 3 .

Claim 4. Every countable subset of $T$ is contained in a countable $r$-admissible subset of $T$.

Let $A$ be a countable subset of $T$. Our goal is to find a countable $r$-admissible subset $C$ such that $A \subseteq C$. Consider the projective system

$$
\mathscr{S}_{B}=\left\{\prod\left\{B_{t}: t \in C\right\}, \pi_{C}^{C^{\prime}}, \exp _{\omega} T\right\}
$$

representing the product $B$ and associated to the subalgebra $X$ projective system

$$
\mathscr{S}_{X}=\left\{\operatorname{cl}_{\prod\left\{B_{t}: t \in C\right\}}\left(\pi_{C}(X)\right), \pi_{C}^{C^{\prime}} \mid \operatorname{cl}_{\prod\left\{B_{t}: t \in C^{\prime}\right\}}\left(\pi_{C^{\prime}}(X)\right), C \in \exp _{\omega} T\right\}
$$

representing $X$. 


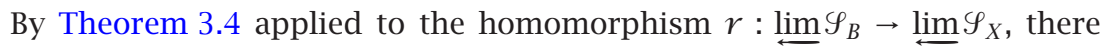
exist a countable subset $C$ of $T$ and a continuous homomorphism $r_{C}: B_{C} \rightarrow$ $\mathrm{cl}_{B_{C}}\left(\pi_{C}(X)\right)$ such that $A \subseteq C$ (i.e., $A \leq C$ in the natural order on $\exp _{\omega} T$ ) and $\pi_{C} \circ r=r_{C} \circ \pi_{C}$. Consider a point $y \in \pi_{C}(X)$. Also pick a point $x \in X$ such that $\pi_{C}(x)=y$. Then,

$$
y=\pi_{C}(x)=\pi_{C}(r(x))=r_{C}\left(\pi_{C}(x)\right)=r_{C}(y)
$$

This shows that $r_{C} \mid \pi_{C}(X)=\operatorname{id}_{\pi_{C}(X)}$. It also follows that $\pi_{C}(X)$ is closed in $B_{C}$.

In order to show that $C$ is $r$-admissible, we consider a point $z \in \pi_{C}^{-1}\left(\pi_{C}(X)\right)$. By the observation made above, $r_{C}\left(\pi_{C}(z)\right)=\pi_{C}(z)$. Finally,

$$
\pi_{C}(z)=r_{C}\left(\pi_{C}(z)\right)=\pi_{C}(r(x))
$$

which implies that $C$ is $r$-admissible.

Since $|T|=\tau$, we can write $T=\left\{t_{\alpha}: \alpha<\tau\right\}$. Since the collection of countable $r$-admissible subsets of $T$ is cofinal in $\exp _{\omega} T$ (see Claim 4), each element $t_{\alpha} \in T$ is contained in a countable $r$-admissible subset $A_{\alpha} \subseteq T$. According to Claim 1, the set $T_{\alpha}=\bigcup\left\{A_{\beta}: \beta \leq \alpha\right\}$ is $r$-admissible for each $\alpha<\tau$. Consider the projective system

$$
\mathscr{S}=\left\{X_{\alpha}, p_{\alpha}^{\alpha+1}, \alpha<\tau\right\}
$$

where

$$
X_{\alpha}=\pi_{T_{\alpha}}(X), \quad p_{\alpha}^{\alpha+1}=\pi_{T_{\alpha}}^{T_{\alpha+1}} \mid \pi_{T_{\alpha+1}}(X) \quad \text { for each } \alpha<\tau
$$

Since $T=\bigcup\left\{T_{\alpha}: \alpha<\tau\right\}$, it follows that $X=\underline{\lim } \mathscr{S}$. Obvious transfinite induction based on Claim 3 shows that

$$
X=\varliminf_{\lim } \mathscr{Y}=X_{0} \times \prod\left\{\operatorname{ker}\left(p_{\alpha}^{\alpha+1}\right): \alpha<\tau\right\} .
$$

In order to demonstrate this, consider the well-ordered projective system $\mathscr{S}^{\prime}=$ $\left\{Z_{\alpha}, q_{\alpha}^{\alpha+1}, \alpha<\tau\right\}$ where

$$
Z_{\alpha}= \begin{cases}X_{0}, & \text { if } \alpha=0, \\ X_{0} \times \prod\left\{\operatorname{ker}\left(p_{\beta}^{\beta+1}\right): \beta<\alpha\right\}, & \text { if } 0<\alpha<\tau\end{cases}
$$

and, for $0<\alpha<\tau$,

$$
q_{\alpha}^{\alpha+1}: X_{0} \times \prod\left\{\operatorname{ker}\left(p_{\beta}^{\beta+1}\right): \beta<\alpha+1\right\} \longrightarrow X_{0} \times \prod\left\{\operatorname{ker}\left(p_{\beta}^{\beta+1}\right): \beta<\alpha\right\}
$$


denotes the natural projection (which forgets the last coordinate). For each $\alpha<\tau$, we construct a topological isomorphism $h_{\alpha}: X_{\alpha} \rightarrow Z_{\alpha}$ as follows. We start by letting $h_{0}=\mathrm{id}_{X_{0}}$. Suppose that topological isomorophism $h_{\gamma}: X_{\gamma} \rightarrow Z_{\gamma}$ has already been constructed for each $\gamma<\alpha$ in such a way that $q_{\gamma}^{\gamma+1} \circ h_{\gamma+1}=$ $h_{\gamma} \circ p_{\gamma}^{\gamma+1}$ whenever $\gamma+1 \leq \alpha$. We construct a topological isomorphism $h_{\alpha}$ : $X_{\alpha} \rightarrow Z_{\alpha}$.

If $\alpha$ is the limit ordinal number, let $h_{\alpha}=\lim \left\{h_{\gamma}: \gamma<\alpha\right\}$.

If $\alpha=\gamma+1$ for some $\gamma<\tau$, then, by Claim 3, there exists a topological homomorphism $h: X_{\gamma+1} \rightarrow X_{\gamma} \times \operatorname{ker}\left(p_{\gamma}^{\gamma+1}\right)$ such that the following diagram

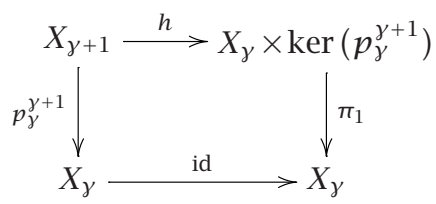

commutes ( $\pi_{1}$ denotes the natural projection onto the first coordinate space). Then, the required topological homomorphism $h_{\gamma+1}: X_{\gamma+1} \rightarrow Z_{\gamma+1}$ is defined as the composition $h_{y+1}=h \circ\left(h_{\gamma} \times\right.$ id). The following diagrams illustrates the situation

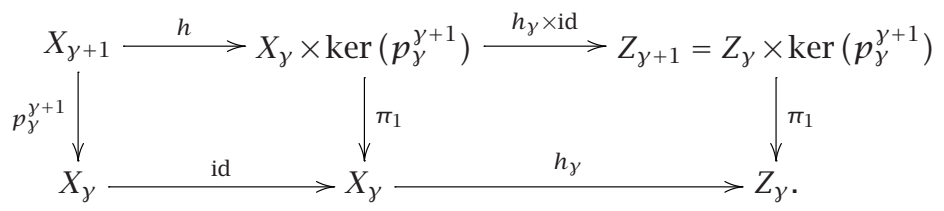

This completes the inductive construction of topological isomorphisms $h_{\alpha}$, $\alpha<\tau$. Finally note that

$$
\begin{aligned}
H & =\lim \left\{h_{\alpha}: \alpha<\tau\right\}: X=\longleftarrow_{\lim } \mathscr{S} \longrightarrow \longleftarrow_{\lim } \mathscr{S}^{\prime} \\
& =X_{0} \times \prod\left\{\operatorname{ker}\left(p_{\alpha}^{\alpha+1}\right): \alpha<\tau\right\}
\end{aligned}
$$

provides the required topological isomorphism.

Since, by the construction, $A_{\alpha}$ is a countable $r$-admissible subset of $T$, it follows from Claim 2 that $X_{0}$ and $\operatorname{ker}\left(p_{\alpha}^{\alpha+1}\right), \alpha<\tau$, are Fréchet algebras. This finishes the proof of Theorem 4.2.

ACKNOWLEDGMENT. This work was partially supported by NSERC research grant.

\section{REFERENCES}

[1] A. Chigogidze, Inverse Spectra, North-Holland Mathematical Library, vol. 53, North-Holland Publishing, Amsterdam, 1996.

[2] _ Complemented subspaces of products of Banach spaces, Proc. Amer. Math. Soc. 129 (2001), no. 10, 2959-2963. 
[3] A. Ya. Helemskii, Banach and Locally Convex Algebras, Oxford Science Publications, The Clarendon Press Oxford University Press, New York, 1993.

[4] A. P. Robertson and W. J. Robertson, Topological Vector Spaces, Cambridge Tracts in Mathematics and Mathematical Physics, no. 53, Cambridge University Press, New York, 1964.

[5] H. H. Schaefer, Topological Vector Spaces, The Macmillan Company, New York, 1966.

Alex Chigogidze: Department of Mathematics and Statistics, University of Saskatchewan, McLean Hall, 106 Wiggins Road, Saskatoon, Saskatchewan, Canada S7N 5E6

E-mail address: chigogid@math. usask.ca 


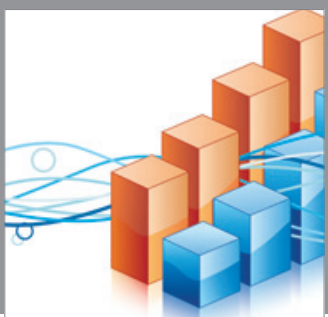

Advances in

Operations Research

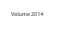

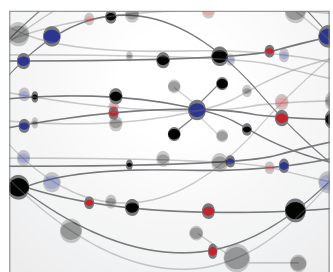

\section{The Scientific} World Journal
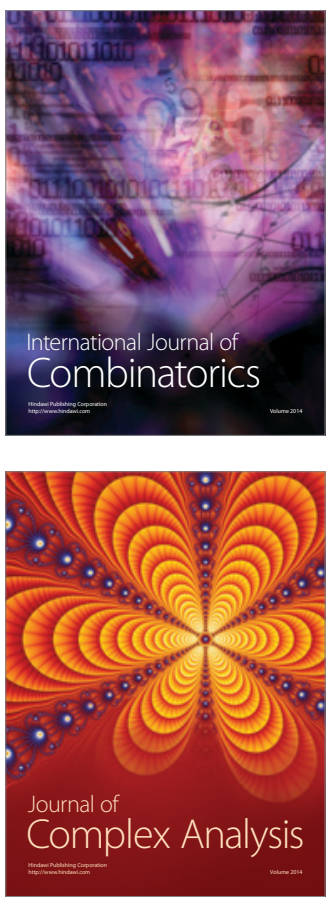

International Journal of

Mathematics and

Mathematical

Sciences
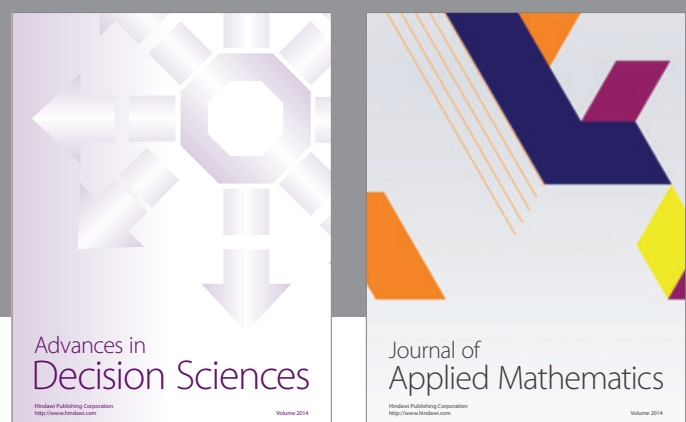

Journal of

Applied Mathematics
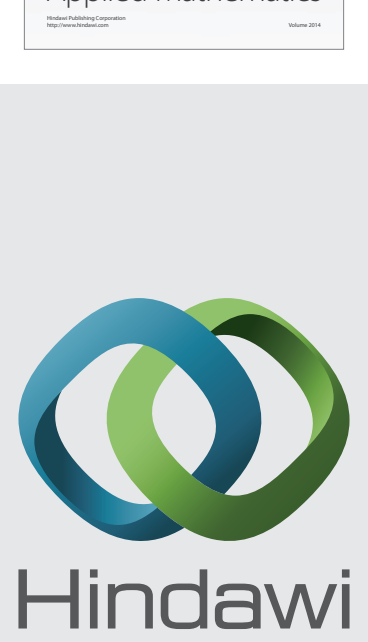

Submit your manuscripts at http://www.hindawi.com
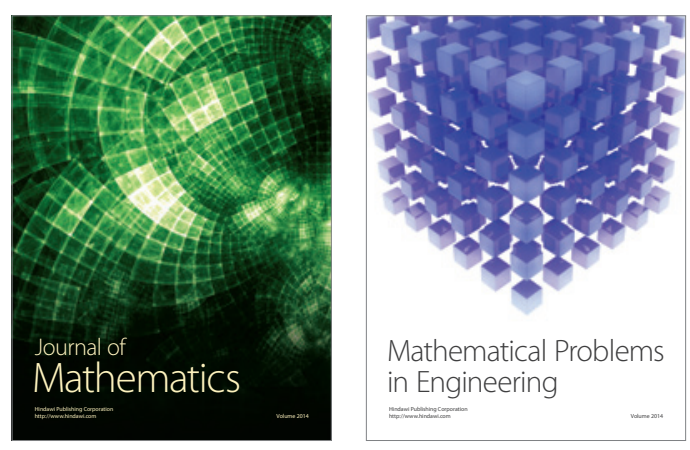

Mathematical Problems in Engineering
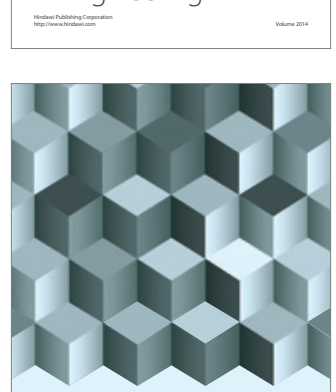

Journal of

Function Spaces
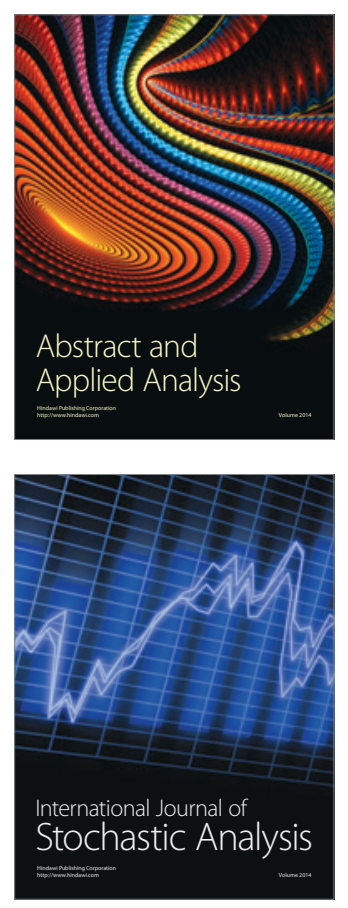

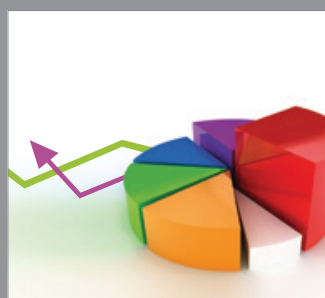

ournal of

Probability and Statistics

Promensencen
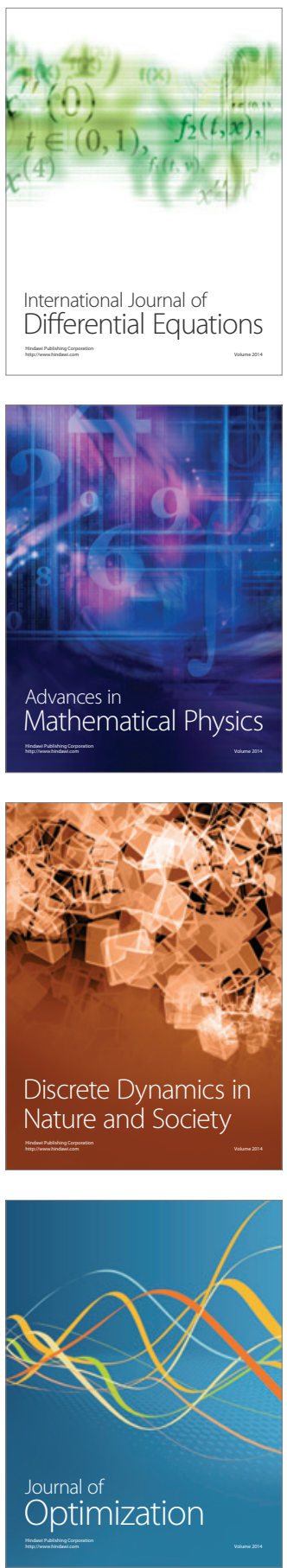